\title{
Effect of tapering on natural frequencies of rotating beams
}

\author{
A. Bazoune \\ Department of Mechanical Engineering, King Fahd University of Petroleum \& Minerals, KFUPM BOX 579, \\ Dhahran 31261, Saudi Arabia \\ E-mail: abazoune@kfupm.edu.sa
}

Received 13 April 2006

Accepted 13 May 2006

\begin{abstract}
The problem of free vibration of a rotating tapered beam is investigated by developing explicit expressions for the mass, elastic and centrifugal stiffness matrices in terms of the taper ratios. This investigation takes into account the effect of tapering in two planes, the effect of hub radius as well as the stiffening effect of rotation. The equations of motion are derived; the associated generalized eigenvalue problem is defined in conjunction with a suitable Lagrangian form and solved for a wide range of parameter changes. The effect of tapering on the natural frequencies of the beam is examined with all parameter changes present. Results are compared with those available in literature and are found to be in excellent agreement.
\end{abstract}

\section{Introduction}

Beams are very common types of structural components. They can be classified according to their geometric properties as uniform or tapered, and slender or thick. Uniform slender beams are widely encountered in literature because of their simple geometry. Taper is desired for optimum distribution of weight and strength and is often motivated by special structural and functional requirements. Rotating beams represent a good model for many engineering structures and find practical applications in aircraft propellers, helicopter blading, high speed flexible mechanisms, robot manipulators and spinning space structures.

The problem of determining the dynamic modal characteristics (natural frequencies and associated mode shapes) of rotating beams is important in design and performance evaluation, and has been the subject of many investigations. Accurate prediction of the dynamic characteristics of such structures is necessary in the early stages of the design process in order to avoid any possible conditions susceptible to resonance within the range of operating speed, and to prevent any failure that may occur as a result of sustained vibration at or near the resonant frequency range.

Rotating structural components have traditionally been modeled as beams vibrating in flexure. Most of the published work devoted to the field of rotating structural components dealing with analytical and numerical methods employs beam models. Many references related to beam vibration problems can be found in published literature and the number of publications is increasing rapidly. Recently, Bazoune [1] presented a thorough review of the several approximate methods used in the bending vibrations of rotating beams.

Compared to rotating uniform beams, tapered Euler-Bernoulli beams have received less attention. Hodges [2] applied the direct analytical method of Ritz to solve for the natural frequencies, mode shapes, and response of a non-uniform rotating beam with discontinuities in bending stiffness and mass distribution. Later, Hodges and Rutkowski [3] presented Legendre polynomial shape functions in conjunction with the FEM to study the natural frequencies of free vibration of rotating beams. In this method, the displacement function is expressed in terms of a complete set of shifted Legendre polynomials. The degree of accuracy depends on the number of elements, $n$, used and the number of terms chosen in the series. Non-dimensional frequency predictions were shown for a non-uniform beam that is linearly tapered in the depth direction only. The fundamental frequency for a tapered beam was shown 
to be higher than that of a prismatic beam whilst the second and third frequencies were shown to be lower than those of the prismatic beam. Wright et al. [4] utilized Hamilton's principle in the formulation of the problem of a rotating beam with a finite hub radius where both flexural rigidity and mass distribution varied in a prescribed manner. A power series solution in the form of the method of Frobenius was employed to obtain estimates for the frequencies and mode shapes. Liu and Yeh [5] used Galerkin's method to predict the bending frequencies of non-uniform rotating Euler-Bernoulli beams with restrained beam base. Yeh and Liu [6] used the Galerkin's method to investigate the influence of taper ratio, elastic root restraints, tip mass, setting angle and rotating speed on the pure bending vibration of rotating non-uniform beams. Storti and Aboelnaga [7] have studied the transverse deflections of a straight tapered symmetric beam attached to a rotating hub as a model for bending vibration of blades in turbomachinery. Wang and Werely [8] extended the work of [2] to rotating tapered beams. They proposed a Spectral Finite Element Method (SFEM). This method consists of determining a solution to the governing differential equation in conjunction with the wave propagation method. Banerjee [9] developed a Dynamic Stiffness Method (DSM) for the determination of the free bending frequencies of an Euler-Bernoulli beam using the Frobenius method of solution.

A review of the current literature shows a lack of formulation for the elemental mass and stiffness matrices for rotating tapered beams as a function of taper ratios. It is the objective of this investigation to develop a finite element procedure for the derivation of the explicit expressions of theses matrices as a function of taper ratios. Next, the differential equations for the free vibration motion are obtained; the generalized eigenvalue problem is defined and solved.

\section{The beam element model}

Figure 1 shows a rotating tapered cantilever beam model. The (XYZ) axes represent a global orthogonal coordinate system with origin at the center of mass of the hub such that the Z-axis corresponds to the spin axis which rotates with a constant angular speed $\Omega$. The (xyz) system is defined as local co-ordinates parallel to the global (XYZ) co-ordinate system and rigidly attached to the root of the beam with its origin shifted by $R_{o}$ from the global (XYZ) co-ordinate system. The $\mathrm{X}$ and $\mathrm{x}$ axes being collinear and coincident with the undeformed beam centerline while the $y$-and z-axes lie along the principal axes of the cross-sectional area of the beam. It is assumed that the center of flexure coincides with the center of mass of the beam so that there is no torsional coupling induced by flexural motion.

The beam configuration can be defined by a properly generated mesh of finite beam elements. These elements are of equal lengths and linearly tapered in two planes while allowing for unequal breadth and depth taper. Each element has a length $l^{i}$ such that the length of the beam is the summation of its individual element lengths. The beam element consists of two nodes, each node undergoes both translational and rotational displacements according to Euler-Bernoulli beam theory. Effects of Coriolis forces are neglected in this investigation.

The transverse displacement $w^{i}$ of the local co-ordinate vector of an arbitrary point $P^{i}$ on element $i$ with respect to the element axes can be expressed as

$$
w^{i}=\mathbf{N}_{1 \times 4}^{i} \mathbf{q}_{4 \times 1}^{i}
$$

where $\mathbf{q}_{4 \times 1}^{i}$ is the vector of nodal coordinates of element $i$ and $\mathbf{N}_{1 \times 4}^{i}$ is the elemental shape functions with non-zero entries given by

$$
\begin{aligned}
& N_{11}^{i}=1-3 \xi^{i 2}+2 \xi^{i 3} \\
& N_{12}^{i}=l^{i}\left(\xi^{i}-2 \xi^{i 2}+\xi^{i 3}\right) \\
& N_{13}^{i}=3 \xi^{i 2}-2 \xi^{33} \\
& N_{14}^{i}=l^{i}\left(\xi^{i 3}-\xi^{i 2}\right)
\end{aligned}
$$

where $\xi^{i}=x^{i} / l^{i}$ is a non-dimensional length. In order to determine the entries of the element matrices, the expression for the breadth and depth of element $i$, are respectively given by

$$
\begin{aligned}
& b\left(\xi^{i}\right)=b_{o}+\left(b-b_{o}\right) \xi^{i}=b_{o}\left(1-\tau_{b} \xi^{i}\right) \\
& h\left(\xi^{i}\right)=h_{o}+\left(h-h_{o}\right) \xi^{i}=h_{o}\left(1-\tau_{h} \xi^{i}\right)
\end{aligned}
$$


where $b_{o}$ and $h_{o}$ are the breadth and depth at the root of the beam. The quantities $\tau_{b}$ and $\tau_{h}$ are the breadth and depth taper ratios, respectively. These are given by

$$
\begin{aligned}
\tau_{b} & =1-\frac{b}{b_{b_{h}}} \\
\tau_{h} & =1-\frac{h}{h_{o}}
\end{aligned}
$$

If $b=b_{o}$ and $h=h_{o}$, the taper ratios vanish and the beam is uniform.

The expression of the elemental cross-section area and the elemental second moment of area are respectively given by

$$
\begin{aligned}
& A^{i}\left(\xi^{i}\right)=A_{o}\left(1-\tau_{b} \xi^{i}\right)\left(1-\tau_{h} \xi^{i}\right) \\
& I^{i}\left(\xi^{i}\right)=I_{o}\left(1-\tau_{b} \xi^{i}\right)\left(1-\tau_{h} \xi^{i}\right)^{3}
\end{aligned}
$$

where $A_{o}=b_{o} h_{o}$ and $I_{o}=(1 / 12) b_{o} h_{o}^{3}$ are the cross-section area and the second moment of area of the cross-section at the root at the beam, respectively.

\section{Derivation of the element matrices}

The strain energy of the $i$ th tapered beam element is given by

$$
U^{i}=\frac{1}{2} \int_{0}^{l^{i}} E I^{i}\left(x^{i}\right)\left(\frac{\partial^{2} w^{i}}{\partial x^{i^{2}}}\right)^{2} \mathrm{~d} x^{i}+\frac{1}{2} \int_{0}^{l^{i}} F_{p}^{i}\left(x^{i}\right)\left(\frac{\partial w^{i}}{\partial x^{i}}\right)^{2} \mathrm{~d} x^{i}
$$

where $E$ represents Young's modulus of elasticity. The first term in Eq. (6) represents the flexural strain energy and the second term the strain energy due to the centrifugal force $F_{p}^{i}\left(x^{i}\right)$. The centrifugal force $F_{p}^{i}\left(x^{i}\right)$ associated with a differential element located at point $P^{i}$ of the element $i$ can be defined as

$$
\mathrm{d} F_{p}^{i}\left(x^{i}\right)=\rho A^{i}\left(x^{i}\right) \Omega^{2}\left(R_{o}+r_{p}^{i}\right) \mathrm{d} r_{p}^{i}
$$

For small deformations one can write

$$
r_{p}^{i}=\left(L_{1}+x^{i}\right)=(m-1) l^{i}+x^{i}
$$

where $m$ and $L_{1}$ are shown in Fig. 1 .

The centrifugal force $F_{p}^{i}\left(x^{i}\right)$ can be obtained by integrating Eq. (7) over the span of the beam between point $P^{i}$ and the free end of the beam. The resulting force $F_{p}^{i}\left(x^{i}\right)$ is then determined and may be expressed as

$$
F_{p}^{i}\left(x^{i}\right)=\rho \Omega^{2} A_{o}\left(\beta_{4} x^{i 4}+\beta_{3} x^{i 3}+\beta_{2} x^{i 2}+\beta_{1} x^{i}+\beta_{o}\right)
$$

where

$$
\begin{aligned}
& \beta_{4}=-\frac{1}{4} l^{i} \tau_{b} \tau_{h} \\
& \beta_{3}=\frac{1}{3 l^{22}}\left[\left(\tau_{b}+\tau_{h}\right) l^{i}-\tau_{b} \tau_{h}\left(L_{1}+R_{o}\right)\right] \\
& \beta_{2}=\frac{1}{2 l^{i}}\left[\left(\tau_{b}+\tau_{h}\right)\left(L_{1}+R_{o}\right)+l^{i}\right] \\
& \beta_{1}=\left(L_{1}+R_{o}\right) \\
& \beta_{0}=\left[\begin{array}{l}
\left(n-L_{1}\right) R_{o} \\
-\frac{1}{12}\left(\tau_{b}+\tau_{h}\right)\left(6\left(n^{2}-m^{2}+1\right) R_{o}+(6 m-2) l^{i}\right) l^{i} \\
+\frac{1}{12} \tau_{b} \tau_{h}\left(4\left(n^{3}-m^{3}+1\right) R_{o}+(4 m-1) l^{i}\right) l^{i} \\
+\frac{1}{12}\left(3 \tau_{b} \tau_{h}\left(n^{4}-m^{4}\right)-4\left(\tau_{b}+\tau_{h}\right)\left(n^{3}-m^{3}\right)+2\left(n^{2}-m^{2}\right)+6(2 m-1)\right) l^{i 2}
\end{array}\right]
\end{aligned}
$$

where $n$ is the total number of elements of the beam. Equation (6) can be written in the form

$$
U^{i}=\frac{1}{2} \mathbf{q}^{i^{T}} \mathbf{k}^{i} \mathbf{q}^{i}
$$




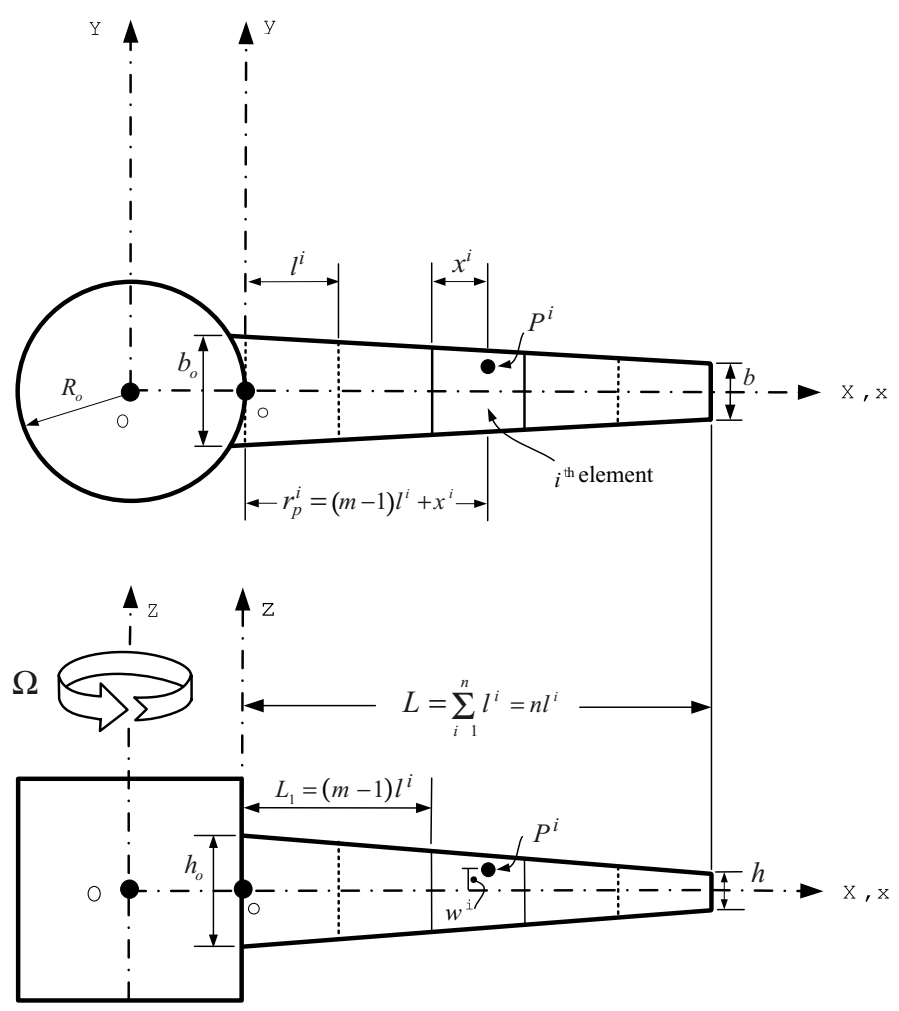

Fig. 1. Rotating cantilever beam linearly tapered in breadth and depth.

where $\mathbf{k}^{i}$ is the composite elemental stiffness matrix given by

$$
\mathbf{k}^{i}=\mathbf{k}_{e}^{i}+\mathbf{k}_{c}^{i}
$$

The matrix $\mathbf{k}_{e}^{i}$ represents the elemental elastic stiffness matrix while the matrix $\mathbf{k}_{c}^{i}$ represents the elemental stiffness matrix due to the contribution of the effect of rotation. These matrices are given by

$$
\begin{aligned}
\mathbf{k}_{e}^{i} & =\int_{0}^{l^{i}}\left[\mathrm{~B}_{e}^{i}\right]^{T} E I^{i}\left(x^{i}\right)\left[\mathrm{B}_{e}^{i}\right] \mathrm{d} x^{i} \\
\mathbf{k}_{c}^{i} & =\int_{0}^{l^{i}}\left[\mathrm{~B}_{c}^{i}\right]^{T} F_{p}^{i}\left(x^{i}\right)\left[\mathrm{B}_{c}^{i}\right] \mathrm{d} x^{i}
\end{aligned}
$$

where

$$
\left[\mathrm{B}_{e}^{i}\right]=\frac{\partial^{2}}{\partial x^{i 2}}\left[\mathbf{N}^{i}\right] \text { and }\left[\mathrm{B}_{c}^{i}\right]=\frac{\partial}{\partial x^{i}}\left[\mathbf{N}^{i}\right]
$$

By carrying out the integration of Eqs (13) and (14), the explicit expressions of the above stiffness matrices are obtained and given in Tables A1 and A2 of the Appendix.

The kinetic energy contribution due to the translational deformation of element $i$ is given by

$$
T^{i}=\frac{1}{2} \dot{\mathbf{q}}^{i^{T}} \mathbf{m}_{e}^{i} \dot{\mathbf{q}}^{i}
$$

where

$$
\mathbf{m}_{e}^{i}=\int_{0}^{l^{i}}\left[\mathbf{N}^{i}\right]^{T} \rho A^{i}\left(x^{i}\right)\left[\mathbf{N}^{i}\right] \mathrm{d} x^{i}
$$


where $\rho$ is the mass density of the beam. By carrying out the integration of Eq. (16), the explicit expression of the mass matrix is obtained and is given in Table A3 of the Appendix.

\section{The generalized eigenvalue problem}

The summation of the individual element energies over the entire beam may be utilized to establish the Lagrangian function $\mathcal{L}$ as

$$
\mathcal{L}=\sum_{i=1}^{n}\left(T^{i}-U^{i}\right)
$$

Substitution of the expression of $\mathcal{L}$ into Lagrange's equation

$$
\frac{\partial}{\partial t}\left(\frac{\partial \mathcal{L}}{\partial \dot{\mathbf{q}}^{i}}\right)-\frac{\partial \mathcal{L}}{\partial \mathbf{q}^{i}}=0
$$

gives the free vibration motion for the rotating tapered beam as

$$
\mathbf{M} \ddot{\mathbf{q}}+\mathbf{K q}=\mathbf{0}
$$

where $\mathbf{q}$ is the vector of all nodal co-ordinates of the beam. The matrices $\mathbf{M}$ and $\mathbf{K}$ are the global mass and stiffness matrices of the entire beam obtained by the standard finite element assembly procedure. Assuming a solution to Eq. (19) of the form

$$
\mathbf{q}=\overline{\mathbf{q}} e^{i \omega t}
$$

where $\overline{\mathbf{q}}$ is a vector of displacement amplitudes of vibration and $\omega$ is the frequency of oscillations. Substitution of Eq. (20) into Eq. (19) yields the generalized eigenvalues problem

$$
\left(\mathbf{K}-\omega^{2} \mathbf{M}\right) \overline{\mathbf{q}}=\mathbf{0}
$$

The solution of Eq. (21) gives the natural frequencies and corresponding mode shapes of a rotating tapered Euler Bernoulli beam.

\section{Results and discussions}

This investigation is concerned with the effect of a doubly linearly tapered beam and takes into account the effects of hub radius and rotation. The beam is linearly tapered in two planes and is divided into 16 equal finite elements. The explicit expressions for the frequency parameter, the rotational speed parameter, and the hub radius parameter are respectively given by

$$
\begin{aligned}
& \bar{\omega}=\frac{\omega_{i}}{\omega_{o}} \\
& \bar{\Omega}=\frac{\Omega}{\omega_{o}} \\
& \bar{R}=\frac{R_{o}}{L}
\end{aligned}
$$

In the above, $L$ represents the total length of the beam and $\omega_{i}$ is the natural frequency of the beam corresponding to the $i$ th mode and $\omega_{o}$ is given by

$$
\omega_{o}=\sqrt{\frac{E I_{o}}{\rho A_{o} L^{4}}}
$$


Table 1

Effect of equal tapering on the frequency parameters of a non-rotating truncated cone cantilever beam with $\bar{R}=0$

\begin{tabular}{clcrrrrr}
\hline Frequency Parameter & $\tau_{b}=\tau_{h}=$ & \multicolumn{1}{c}{0.0} & \multicolumn{1}{c}{0.2} & \multicolumn{1}{c}{0.4} & \multicolumn{1}{c}{0.5} & \multicolumn{1}{c}{0.6} & \multicolumn{1}{c}{0.8} \\
\hline $\bar{\omega}_{1}$ & Present work & 3.5160 & 3.8551 & 4.3188 & 4.6251 & 5.0009 & 6.1964 \\
& Ref. [10] & N/A. & 3.8464 & 4.3174 & 4.6255 & 5.0009 & 6.1963 \\
$\bar{\omega}_{2}$ & Present work & 22.0345 & 21.0571 & 20.0503 & 19.5479 & 19.0653 & 18.3859 \\
& Ref. [10] & N/A. & 21.0569 & 20.0499 & 19.5476 & 19.0649 & 18.3885 \\
$\bar{\omega}_{3}$ & Present work & 61.6772 & 56.6372 & 51.3411 & 48.5853 & 45.7448 & 39.8403 \\
& Ref. [10] & N/A. & 56.6303 & 51.3346 & 48.5789 & 45.7384 & 39.8336 \\
\hline
\end{tabular}

Table 2

The first three frequency parameters of a non-rotating cantilever wedge and cone beam with $\bar{R}=0$

\begin{tabular}{|c|c|c|c|c|c|c|}
\hline & \multicolumn{3}{|c|}{ Cantilever Wedge $\tau_{b}=0.0, \tau_{h}=1.0$} & \multicolumn{3}{|c|}{ Cantilever Cone $\tau_{b}=\tau_{h}=1.0$} \\
\hline & $\bar{\omega}_{1}$ & $\bar{\omega}_{2}$ & $\bar{\omega}_{3}$ & $\bar{\omega}_{1}$ & $\bar{\omega}_{2}$ & $\bar{\omega}_{3}$ \\
\hline Present work & 5.3151 & 15.2074 & 30.0269 & 8.7193 & 21.1465 & 38.4661 \\
\hline Ref. [11] & 5.3151 & 15.2072 & 30.0198 & 8.7193 & 21.1457 & 38.4538 \\
\hline
\end{tabular}

Table 3

Effect of rotation on the frequency parameters of rotating tapered cantilever beam for $\bar{\sigma}=0.0$ and $\tau_{h}=0.5$ and $\bar{R}=0$

\begin{tabular}{|c|c|c|c|c|c|c|}
\hline \multirow{2}{*}{$\begin{array}{c}\text { Rotational speed } \\
\text { parameter } \bar{\Omega}\end{array}$} & \multicolumn{2}{|c|}{$\bar{\omega}_{1}$} & \multicolumn{2}{|c|}{$\bar{\omega}_{2}$} & \multicolumn{2}{|c|}{$\bar{\omega}_{3}$} \\
\hline & Present & Ref. [8] & Present & Ref. [8] & Present & Ref. [8] \\
\hline 0 & 3.8238 & 3.8238 & 18.3173 & 18.3173 & 47.2648 & 47.2648 \\
\hline 1 & 3.9865 & 3.9866 & 18.4739 & 18.4740 & 47.4170 & 47.4173 \\
\hline 2 & 4.4354 & 4.4368 & 18.9358 & 18.9366 & 47.8689 & 47.8716 \\
\hline 3 & 5.0920 & 5.0927 & 19.6800 & 19.6839 & 48.5061 & 48.6190 \\
\hline 4 & 5.8500 & 5.8788 & 20.6671 & 20.6852 & 49.4395 & 49.6456 \\
\hline 5 & 6.7377 & 6.7434 & 21.8745 & 21.9053 & 50.2987 & 50.9338 \\
\hline 6 & 7.6380 & 7.6551 & 23.2800 & 23.3093 & 51.8356 & 52.4633 \\
\hline 7 & 8.5296 & 8.5956 & 24.0234 & 24.8647 & 53.6578 & 54.2124 \\
\hline 8 & 9.5035 & 9.5540 & 25.3245 & 26.5437 & 55.0034 & 56.1595 \\
\hline 9 & 10.3293 & 10.5239 & 27.1023 & 28.3227 & 57.0386 & 58.2833 \\
\hline 10 & 11.2765 & 11.5015 & 28.0945 & 30.1827 & 58.5639 & 60.5639 \\
\hline
\end{tabular}

Table 1 shows the effect of equal tapering on the first three frequency parameters of a non-rotating truncated conical cantilever beam at different taper ratios. The comparison of these frequencies shows an excellent agreement with the numerical values presented by Downs [10] who reported numerical solutions based on Frobenius method. Table 2 shows two particular cases of tapering, namely a wedge and a cone. The results compare favorably with other methods of solutions such as the direct solution method of the mode shape equation used by Naguleswaran [11]. The effect of rotational speed for fixed taper ratios of $\tau_{b}=0$ and $\tau_{b}=0.5$ is shown in Table 3. Results manifested in Table 3 are in good agreement with those of G. Wang and N.M. Wereley [8] who used Spectral Finite Element Method.

The effects of tapering and rotation on the first three frequency parameters of the beam at $R=0$ are shown in Fig. 2. In this figure, the second and third frequency parameters decrease as the taper ratios increase while the fundamental frequency increases. This trend is seen to be opposite to the one manifested in Table 1 for the non-rotating case.

The effect of unequal tapering as well as hub radius parameter changes on the first three frequency parameters are examined next, as manifested in Figs 3 to 5. In these figures, it is shown that as the hub radius parameter increases, the first three frequency parameters increase. In particular, it is noticed in Fig. 3 that at a specific hub radius parameter of $\bar{R}=0$ and at a rotational speed parameter of approximately 3.5, the values of fundamental frequency parameters corresponding to the different taper ratios are equal. This is also repeated for $\bar{R}=0.5$ and $\bar{R}=1.0$. Figures 4 and 5 show that the second and third frequency parameters exhibit a stiffening effect that is due to the increase of the hub on one hand and the hub rotation on the other hand. However, there are cross-over frequencies as a result of combining the effects of taper ratios, rotational speed parameter and the hub radius parameter. 


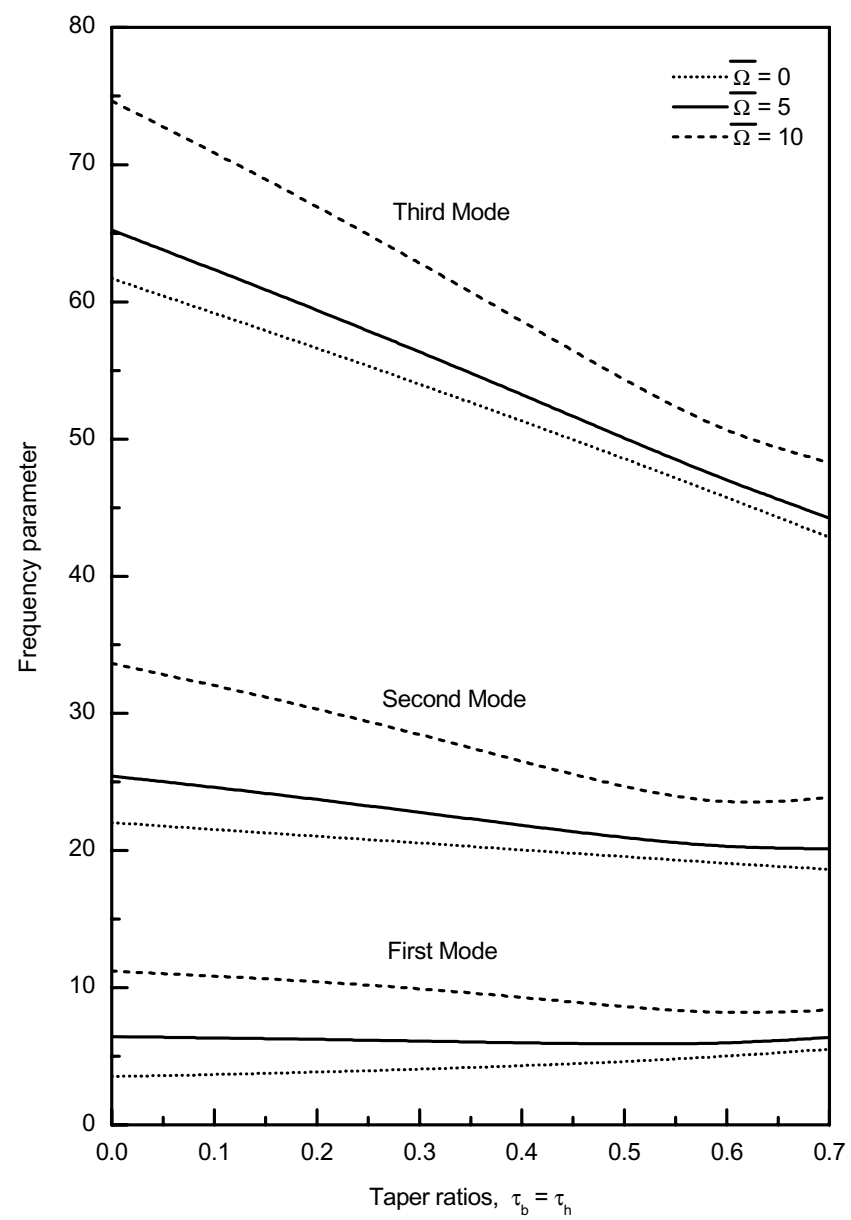

Fig. 2. Effect of equal taper ratios on the frequency parameters of rotating Euler-Bernoulli beam with $\bar{R}=0$.

\section{Conclusion}

The free vibration problem of a rotating tapered beam is investigated by developing explicit expressions of the mass, elastic stiffness and centrifugal stiffness matrices as a function of the taper ratios. As the effect of taper ratios is eliminated from these matrices one can easily obtain the explicit expression of the mass and elastic stiffness matrices that are usually encountered in any elementary finite element text for uniform beams. The taper ratios considered in this problem are linear in depth and breadth. Frequency parameters are shown to be affected by varying both tapers. As the hub radius parameter increases the frequency parameters are seen to increase. In addition, these frequencies exhibit a stiffening effect due to hub rotation. While varying all three parameters together the frequencies show cross over at different speed, taper ratios and hub radius parameters.

\section{Acknowledgment}

The support provided by King Fahd University of Petroleum \& Minerals is deeply appreciated.

\section{Appendix}

Table A1 Bending stiffness matrix of rotating tapered Euler-Bernoulli beam element.

The bending stiffness matrix for tapered Euler-Bernoulli beam is given by 


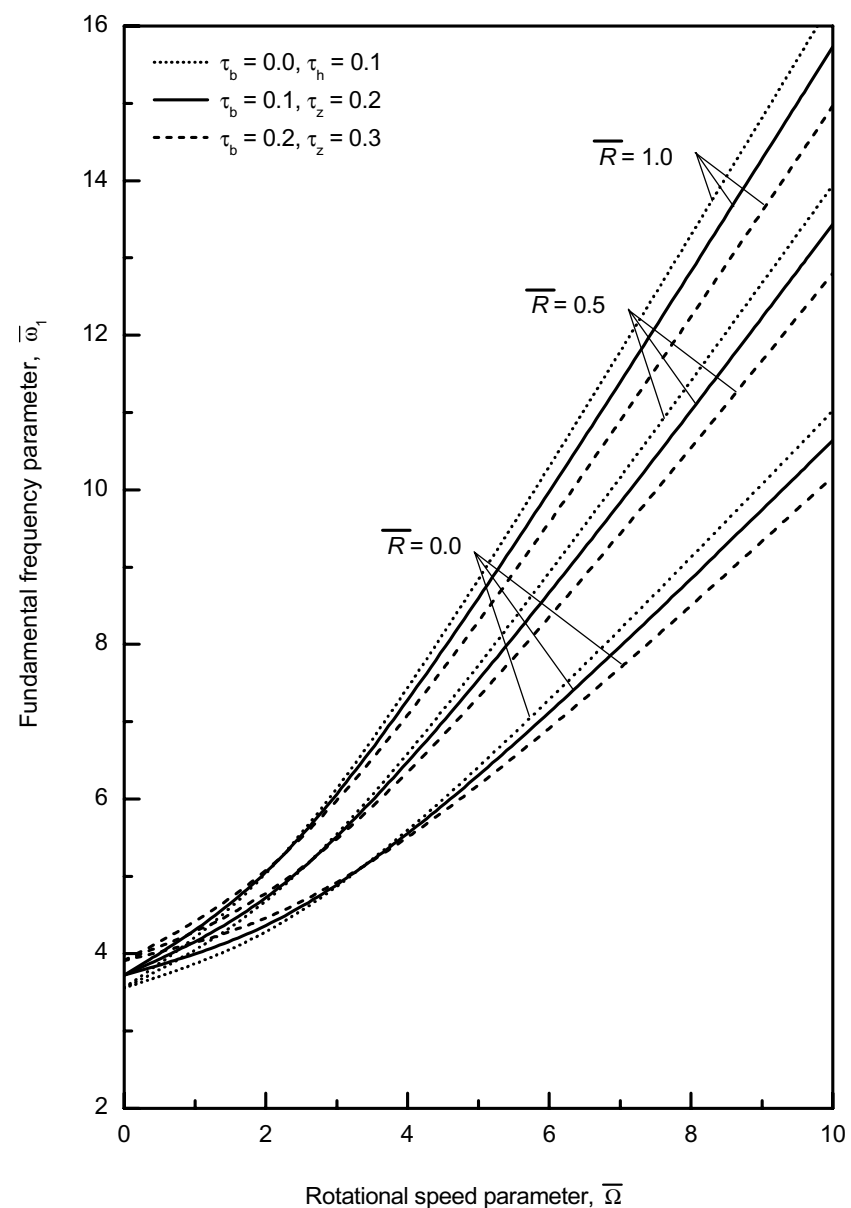

Fig. 3. Effect of unequal tapering on the fundamental frequency parameter of rotating Euler-Bernoulli beam.

$$
\overline{\mathbf{k}_{e}^{i}=\frac{E I_{o}}{l^{i^{3}}}\left[k_{i, j}^{e}\right], \quad i, j=1, \ldots, 4}
$$

where the elements of the lower triangular part of the bending stiffness matrix $\left[k_{i, j}^{e}\right]$ are given by

$$
\begin{aligned}
& k_{1,1}^{e}=\frac{3}{35}\left(44 \tau_{b} \tau_{h}^{3}-49 \tau_{h}^{3}-147 \tau_{b} \tau_{h}^{2}+168 \tau_{h}^{2}+168 \tau_{b} \tau_{h}-210 \tau_{h}-70 \tau_{b}+140\right) \\
& k_{2,1}^{e}=\left(-\frac{1}{70}\right)\left(-76 \tau_{b} \tau_{h}^{3}+84 \tau_{h}^{3}+252 \tau_{b} \tau_{h}^{2}-294 \tau_{h}^{2}-294 \tau_{b} \tau_{h}+420 \tau_{h}+140 \tau_{b}-420\right) l^{i} \\
& k_{3,1}^{e}=-k_{1,1}^{e} \\
& k_{4,1}^{e}=\frac{1}{70}\left(188 \tau_{b} \tau_{h}^{3}-210 \tau_{h}^{3}-630 \tau_{b} \tau_{h}^{2}+714 \tau_{h}^{2}+714 \tau_{b} \tau_{h}-840 \tau_{h}-280 \tau_{b}+420\right) l^{i} \\
& k_{2,2}^{e}=\frac{1}{140}\left(48 \tau_{b} \tau_{h}^{3}-56 \tau_{h}^{3}-168 \tau_{b} \tau_{h}^{2}+224 \tau_{h}^{2}+224 \tau_{b} \tau_{h}-420 \tau_{h}-140 \tau_{b}+560\right) l^{i^{2}} \\
& k_{3,2}^{e}=-k_{2,1}^{e}
\end{aligned}
$$




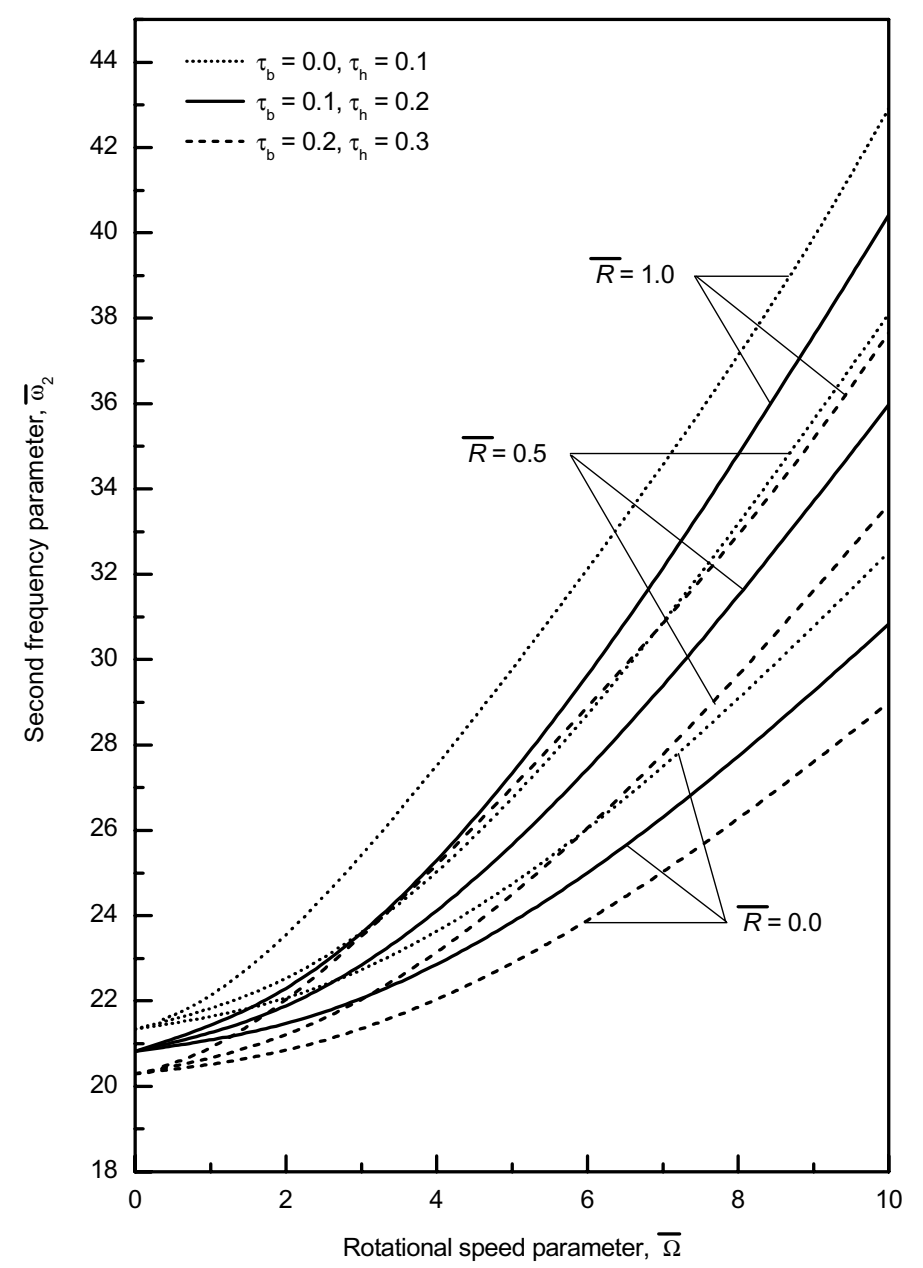

Fig. 4. Effect of unequal tapering on the second frequency parameter of rotating Euler-Bernoulli beam.

$$
\begin{aligned}
& k_{4,2}^{e}=-\frac{1}{140}\left(-104 \tau_{b} \tau_{h}^{3}+112 \tau_{h}^{3}+336 \tau_{b} \tau_{h}^{2}-364 \tau_{h}^{2}-364 \tau_{b} \tau_{h}+420 \tau_{h}+140 \tau_{b}-280\right) l^{i^{2}} \\
& k_{3,3}^{e}=k_{1,1}^{e} \\
& k_{4,3}^{e}=-k_{4,1}^{e} \\
& k_{4,4}^{e}=\frac{1}{140}\left(272 \tau_{b} \tau_{h}^{3}-308 \tau_{h}^{3}-924 \tau_{b} \tau_{h}^{2}-1064 \tau_{h}^{2}-1064 \tau_{b} \tau_{h}-1260 \tau_{h}-420 \tau_{b}+560\right) l^{i^{2}}
\end{aligned}
$$

Table A2 Centrifugal stiffness matrix of rotating tapered Euler-Bernoulli beam element.

The centrifugal stiffness matrix for tapered Euler-Bernoulli beam is given by

$$
\overline{\mathbf{k}_{c}^{i}=\frac{\rho \Omega^{2} A_{o}}{l^{i}}\left[k_{i, j}^{c}\right], \quad i, j=1, \ldots, 4}
$$

where the elements of the lower triangular part of the centrifugal stiffness matrix $\left[k_{i, j}^{c}\right]$ are given by

$$
k_{1,1}^{c}=\frac{1}{420}\left(504 \beta_{0}+252 \beta_{1} l^{i}+144 \beta_{2} l^{i^{2}}+90 \beta_{3} l^{i^{3}}+604 \beta_{4} l^{i^{4}}\right)
$$




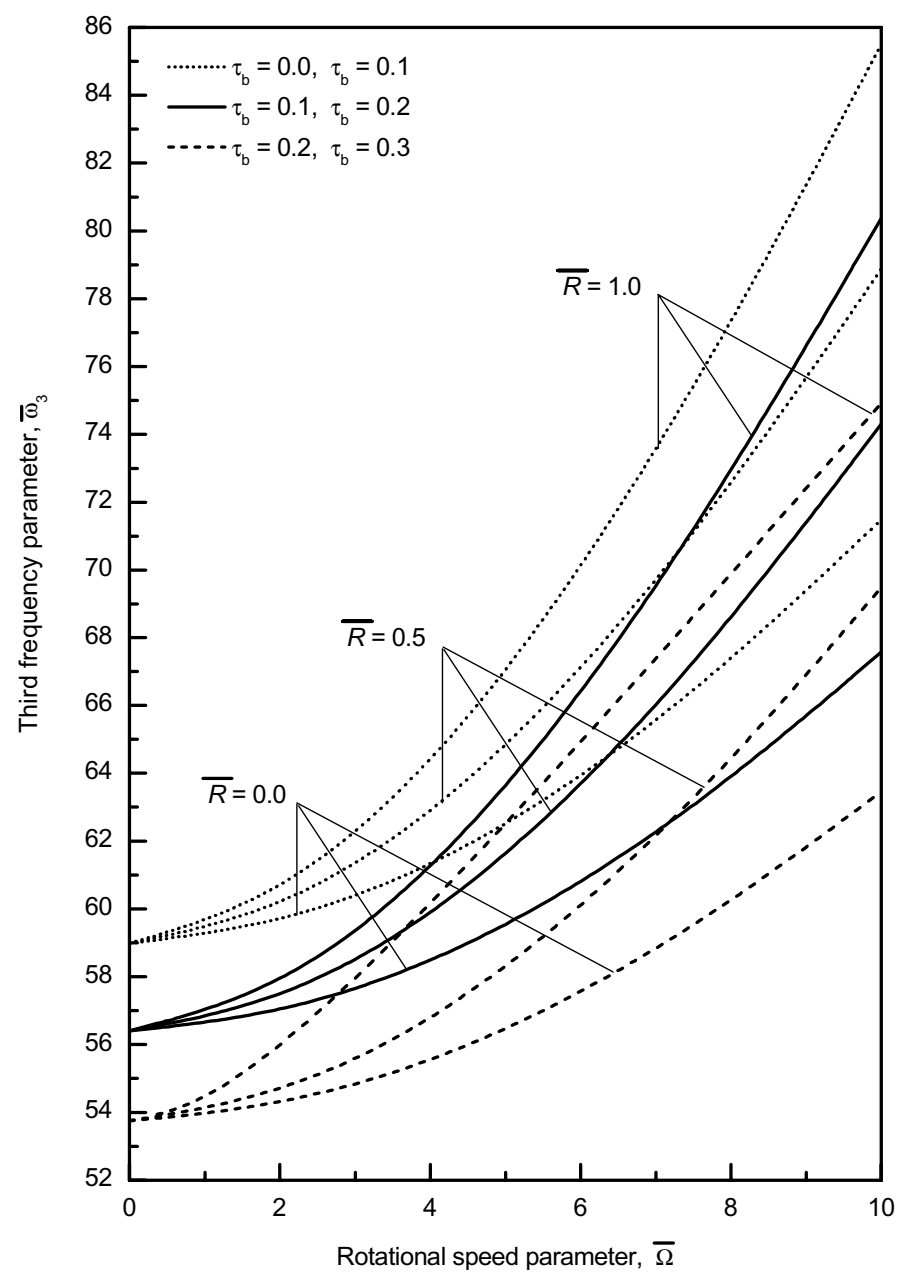

Fig. 5. Effect of unequal tapering on the third frequency parameter of rotating Euler-Bernoulli beam.

$$
\begin{aligned}
& k_{2,1}^{c}=\frac{1}{840}\left(84 \beta_{0}+84 \beta_{1} l^{i}+60 \beta_{2} l^{i^{2}}+42 \beta_{3} l^{i^{3}}+30 \beta_{4} l^{i^{4}}\right) l^{i} \\
& k_{3,1}^{c}=-k_{1,1}^{c} \\
& k_{4,1}^{c}=-\frac{1}{840}\left(-84 \beta_{0}+24 \beta_{2} l^{i^{2}}+30 \beta_{3} l^{3^{3}}+30 \beta_{4} l^{i^{4}}\right) l^{i} \\
& k_{2,2}^{c}=\frac{1}{1680}\left(224 \beta_{0}+56 \beta_{1} l^{i}+32 \beta_{2} l^{i^{2}}+22 \beta_{3} l^{i^{3}}+16 \beta_{4} l^{i^{4}}\right) l^{i 2} \\
& k_{3,2}^{c}=-k_{2,1}^{c} \\
& k_{4,2}^{c}=-\frac{1}{1680}\left(56 \beta_{0}+28 \beta_{1} l^{i}+24 \beta_{2} l^{i^{2}}+22 \beta_{3} l^{i^{3}}+20 \beta_{4} l^{i^{4}}\right) l^{i^{2}}
\end{aligned}
$$




$$
\begin{aligned}
& k_{3,3}^{c}=k_{1,1}^{c} \\
& k_{4,3}^{c}=-k_{4,1}^{c} \\
& k_{4,4}^{w}=\frac{1}{1680}\left(224 \beta_{0}+168 \beta_{1} l^{i}+144 \beta_{2} l^{i^{2}}+130 \beta_{3} l^{i^{3}}+120 \beta_{4} l^{i^{4}}\right) l^{i^{2}}
\end{aligned}
$$

Table A3 Translational mass matrix of rotating tapered Euler-Bernoulli beam element The mass matrix for tapered Euler-Bernoulli beam is given by

$$
\overline{\mathbf{m}_{e}^{i}=\frac{\rho A_{o} l^{i}}{2520}\left[m_{i, j}^{e}\right], \quad i, j=1, \ldots, 4}
$$

where the elements of the lower triangular part of the translational mass matrix $\left[\mathbf{m}_{i, j}^{w}\right]$ are given by

$$
\begin{aligned}
& m_{1,1}^{e}=76 \tau_{b} \tau_{h}^{2}-216 \tau_{h}-216 \tau_{b}+936 \\
& m_{2,1}^{e}=\left(17 \tau_{b} \tau_{h}^{2}-42 \tau_{h}-42 \tau_{b}+132\right) l^{i} \\
& m_{3,1}^{e}=92 \tau_{b} \tau_{h}^{2}-162 \tau_{h}-162 \tau_{b}+324 \\
& m_{4,1}^{e}=-\left(19 \tau_{b} \tau_{h}^{2}-36 \tau_{h}-36 \tau_{b}+78\right) l^{i} \\
& m_{2,2}^{e}=\left(4 \tau_{b} \tau_{h}^{2}-9 \tau_{h}-9 \tau_{b}+24\right) l^{i^{2}} \\
& m_{3,2}^{e}=\left(25 \tau_{b} \tau_{h}^{2}-42 \tau_{h}-42 \tau_{b}+78\right) l^{i} \\
& m_{4,2}^{e}=-\left(5 \tau_{b} \tau_{h}^{2}-9 \tau_{h}-9 \tau_{b}+18\right) l^{i^{2}} \\
& m_{3,3}^{e}=580 \tau_{b} \tau_{h}^{2}-720 \tau_{h}-720 \tau_{b}+936 \\
& m_{4,3}^{e}=-\left(65 \tau_{b} \tau_{h}^{2}-90 \tau_{h}-90 \tau_{b}+132\right) l^{i} \\
& m_{4,4}^{e}=-\left(10 \tau_{b} \tau_{h}^{2}-15 \tau_{h}-15 \tau_{b}+24\right) l^{i^{2}}
\end{aligned}
$$

\section{References}

[1] A. Bazoune, Survey On Modal Frequencies of Centrifugally Stiffened Beams, Shock and Vibration Digest 37(6) (2005), 449-469.

[2] D.H. Hodges, Vibration and Response of Non-uniform Rotating Beams with Discontinuities, Journal of the American Helicopter Society 24 (1979), 43-50.

[3] D.H. Hodges and M.J. Rutkowski, Free Vibration Analysis of Rotating Beams by a Variable-Order Finite-Element Method, AIAA Journal 19 (1981), 1459-1466.

[4] A.D. Wright, C.E. Smith, R.W. Thresher and J.L.C. Wang, Vibration Modes of Centrifugally Stiffened Beams, Transactions of the ASME, Journal of Applied Mechanics 49 (1982), 197-202.

[5] W.H. Liu and F.H. Yeh, Vibrations of Non-uniform Rotating Beams, Journal of Sound and Vibration 119 (1987), $379-384$.

[6] Y. Liu, F.H. Yeh and W.H. Liu, Transverse Vibrations of Non-uniform Rotating Beams with Rotational and Translational Restraints, Journal of the Chinese Society of Mechanical Engineers 10 (1989), 393-400.

[7] D. Storti and Y. Aboelnaga, Bending Vibrations of a Class of Rotating Beams with Hypergeometric Solutions, Transactions of the ASME, Journal of Applied Mechanics 54 (1987), 311-314.

[8] G. Wang and N.M. Wereley, Free Vibration Analysis of Rotating Blades with Uniform Tapers, AIAA Journal 42 (2004), $2429-2437$.

[9] J.R. Banerjee, Free Vibration of Centrifugally Stiffened Uniform and Tapered Beams Using the Dynamic Stiffness Method, Journal of Sound and Vibration 233 (2000), 857-875.

[10] B. Downs, Reference Frequencies For The Validation of Numerical Solutions of Transverse Vibrations on Non-Uniform Beams, Journal of Sound and Vibration 61(1) (1978), 71-78.

[11] S. Naguleswaran, A Direct Solution of the Transverse Vibration of Euler-Bernoulli Wedge and Cone Beams, Journal of Sound and Vibration 172(3) (1994), 289-304. 

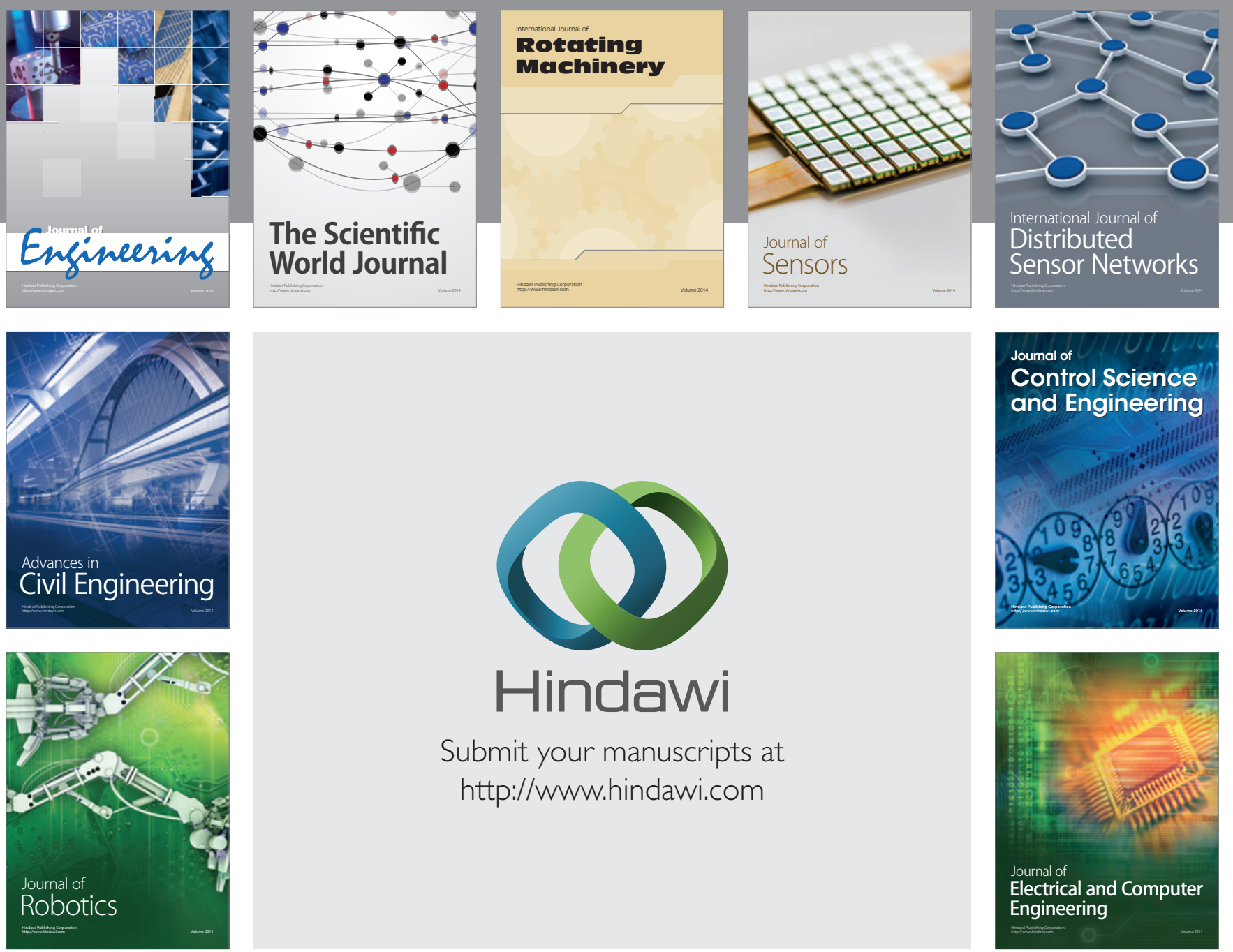

Submit your manuscripts at

http://www.hindawi.com
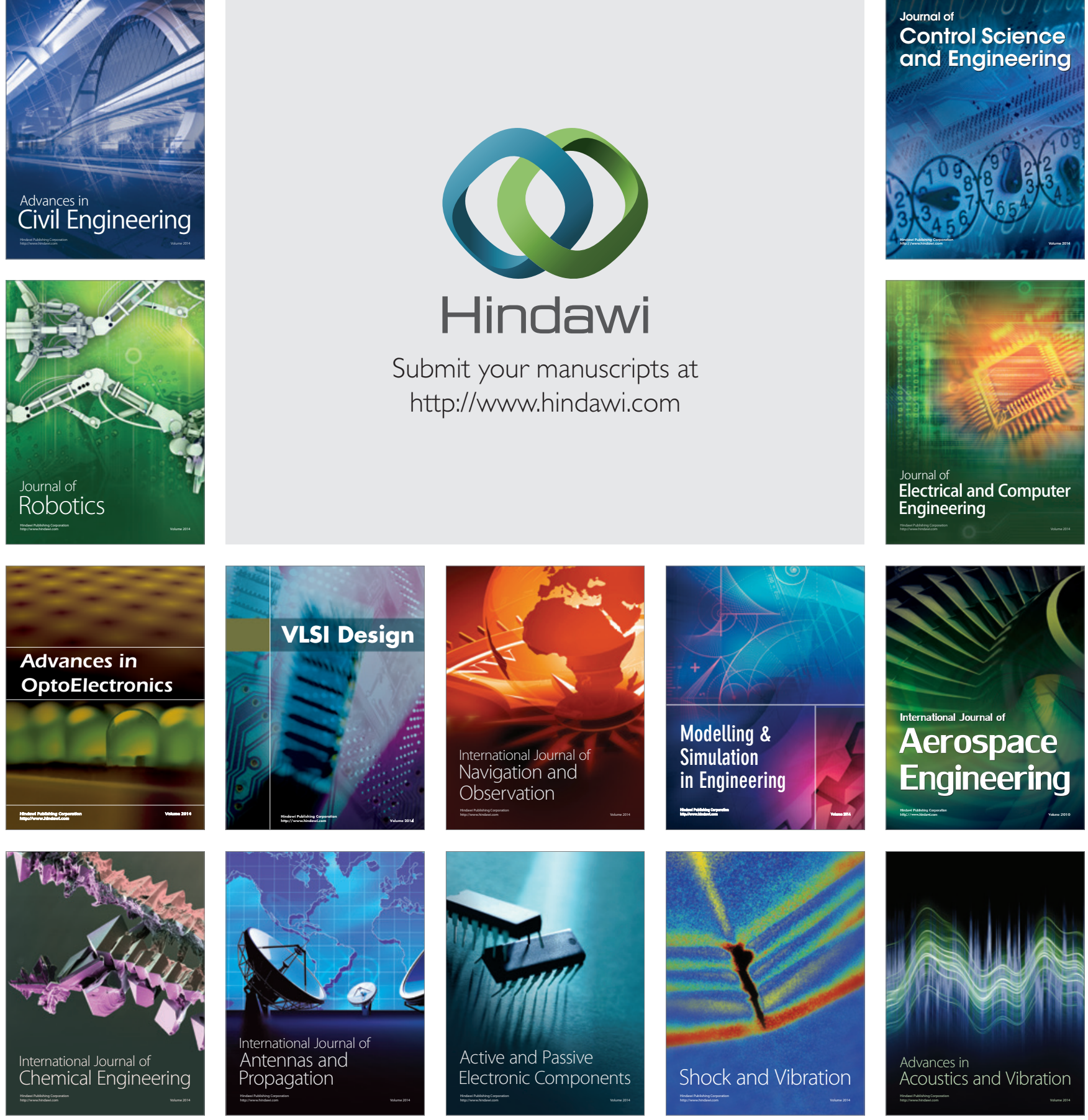